\title{
THE CENTRALIZER OF THE LAGUERRE POLYNOMIAL SET
}

\author{
N.A. AL-SALAM AND W.A. AL-SALAM
}

1. Introduction. By a polynomial set (p.s.) we mean a sequence $P=$ $\left\{P_{0}(x), P_{1}(x), P_{2}(x), \cdots\right\}$ of polynomials in which $P_{0}(x) \neq 0$ and $P_{n}(x)$ is of exact degree $n$. In this work we shall be interested in sets (or classes) whose elements are themselves polynomial sets. This point of view is not new. Appell [2] considered the class $\mathscr{A}$ of Appell polynomials $A=\left\{A_{n}(x)\right\}$ whose generating function is

$$
A(t) e^{x t}=\sum_{n=0}^{\infty} A_{n}(x) \frac{t^{n}}{n !} .
$$

The Sheffer class $\mathscr{S}[6]$ is the class of all p.s. $S=\left\{S_{n}(x)\right\}$ for which

$$
A(t) e^{x H(t)}=\sum_{n=0}^{\infty} S_{n}(x) \frac{t^{n}}{n !} .
$$

Similarly the Boas-Buck class $\mathscr{B}$ consists of all p.s. $B$ for which [3]

$$
A(t) \Phi(x H(t))=\sum_{n=0}^{\infty} \phi_{n} B_{n}(x) t^{n},
$$

where in these formulas $A(t), H(t)$ and $\Phi(t)$ are formal power series such that $A(0) \neq 0, H(0)=0$ but $H^{\prime}(0) \neq 0$, and $\Phi(t)=\phi_{0}+\phi_{1} t+\phi_{2} t^{2}+$ ... with $\phi_{k} \neq 0$ for all $k \geqq 0$. (1.1) is obtained when $H(t)=t$ and $\Phi(t)=$ $e^{t}$

Many of the well known p.s. are included in one or more of the above classes. For example, the Hermite p.s. is in $\mathscr{A}$ as well as in $\mathscr{S}$. The Laguerre p.s. $L^{(\alpha)}$ is in $\mathscr{S}$. Other examples are the Abel, the Meixner, the Bernoulli, and the Boole polynomial sets.

Appell [2], Sheffer [6] as well as Rota, Kahaner and Odlysko [5] (see also [4]) gave sets of polynomials ( $\mathscr{A}$ in [2], $\mathscr{S}$ in [4], [5], [6]) an algebraic structure by defining multiplication in the following manner.

Let $P=\left\{P_{n}(x)\right\}$ and $Q=\left\{Q_{n}(x)\right\}$ be two elements of the set under consideration. Let, furthermore, $P_{n}(x)=\sum_{k=0}^{n} p_{n k} x^{k}$ and $Q_{n}(x)=$ 
$\sum_{k=0}^{n} q_{n k} x^{k}$ for all $n$. Then the (umbral) product $R \equiv P Q$ is defined as the p.s. for which $R_{n}(x)=P_{n}(Q)=\sum_{k=0}^{n} p_{n k} Q_{k}(x) n=0,1,2, \ldots$.

It is clear that $\pi$, the set of all p.s., with this multiplication is a group (non-commutative) in which the identity is $I=\left\{x^{n}, n=0,1,2, \ldots\right\}$.

In [1] the present authors characterized the centralizer, $C_{\mathscr{B}}\left(L^{(\alpha)}\right)$, of the Laguerre p.s. in the Boas-Buck group $\mathscr{B}$.

If we recall that $\mathscr{B} \subset \pi$ it becomes natural to characterize elements of $C_{\pi}\left(L^{(\alpha)}\right)$ the centralizer of $L^{(\alpha)}$ in $\pi$.

As we shall see that, perhaps due to the fact that $\pi$ lacks the nice structure that $\mathscr{B}$ has, this problem is somewhat more difficult than the problem considered in [1]. To our surprise the Euler numbers and polynomials played a prominent role in the solution (which did not arise in [1]).

2. Preliminaries. Let us recall the Euler polynomials

$$
\frac{2}{e^{t}+1} e^{x t}=\sum_{n=0}^{\infty} E_{n}(x) \frac{t^{n}}{n !}
$$

and the related tangent numbers $C_{0}=1$ and

$$
\tanh t=-\sum_{n=1}^{\infty} C_{n} \frac{t^{n}}{n !}
$$

so that $C_{2 n}=0$ if $n>0$ and $C_{2 n+1}=2^{2 n+1} E_{2 n+1}(0)$. We shall abbreviate $C_{2 n+1} / 2^{2 n+1}$ by $(-1)^{n-1} \alpha_{n}(n=0,1,2, \ldots)$.

It follows that in term of the Bernoulli numbers we have $C_{n}=1+$ $2^{n}\left(1-2^{n}\right) B_{n} / n(n \geqq 1)$, and that

$$
C_{n}+(2+C)^{n}= \begin{cases}0 & (n>0) \\ 2 & (n=0)\end{cases}
$$

and

$$
x^{n}=\frac{1}{2}\left\{E_{n}(x+1)+E_{n}(x)\right\} .
$$

In (2.3) $(2+C)^{n}$ is to be expanded by the binomial theorem and $C^{k}$ be replaced by $C_{k}$.

In this work we shall need the following lemmas:

LEMma 1. We have for $N=1,2,3, \ldots$

$$
\begin{gathered}
\alpha_{N}=-\frac{1}{2}\left\{1+\sum_{j=0}^{N-1}(-1)^{j-1} \alpha_{j}\left(\begin{array}{c}
2 N+1 \\
2 j+1
\end{array}\right)\right\}, \\
-1=\sum_{j=0}^{N-1}(-1)^{j-1} \alpha_{j}\left(\begin{array}{l}
2 N \\
2 j+1
\end{array}\right) .
\end{gathered}
$$


These formulas follow from (2.3) with $n=2 N+1$ and $n=2 N$ respectively.

LeMma 2. For $0 \leqq r \leqq 2 m+1$ we have

$$
-\frac{1}{2} \sum_{k \geqq 0}\left\{(-1)^{k}\left(\begin{array}{c}
2 m+1-r \\
k
\end{array}\right)+\left(\begin{array}{l}
r \\
k
\end{array}\right)\right\} \frac{C_{2 m+1-k}}{2^{2 m+1-k}}=\delta_{0 r} .
$$

LeMma 3. For $0 \leqq r \leqq 2 m$ we have

$$
\sum_{k \geq 0}\left\{\left(\begin{array}{c}
r \\
2 k+1
\end{array}\right)-\left(\begin{array}{c}
2 m-r \\
2 k+1
\end{array}\right)\right\} \frac{C_{2 m-2 k-1}}{2^{2 m-2 k-1}}=\delta_{0 r} .
$$

To prove (2.6) and (2.7) let $f(x)$ be the polynomial defined by

$$
f(x)=x^{r}(x-1)^{m-r} \quad(0 \leqq r \leqq m) .
$$

Then

$$
f(x)+f(x+1)=\sum_{k \geq 0}\left\{(-1)^{k}\left(\begin{array}{c}
m-r \\
k
\end{array}\right)+\left(\begin{array}{l}
r \\
k
\end{array}\right)\right\} x^{m-k} .
$$

This, using (2.4), gives

$$
f(x)+f(x+1)=\frac{1}{2} \sum_{k \geq 0}\left\{(-1)^{k}\left(\begin{array}{c}
m-r \\
k
\end{array}\right)+\left(\begin{array}{c}
r \\
k
\end{array}\right)\right\}\left\{E_{m-k}(x)+E_{m-k}(x+1)\right\} .
$$

But if $g(x)$ is a polynomial such that $g(x)+g(x+1) \equiv 0$ then $g(x) \equiv 0$. Hence we get

$$
f(x) \equiv \frac{1}{2} \sum_{k \geq 0}\left\{(-1)^{k}\left(\begin{array}{c}
m-r \\
k
\end{array}\right)+\left(\begin{array}{c}
r \\
k
\end{array}\right)\right\} E_{m-k}(x) .
$$

Now putting $m$ even or odd and $x=0$ we get either (2.7) or (2.6).

3. The Centralizer $\mathbf{C}_{\pi}\left(\mathbf{L}^{(\alpha)}\right)$. Let $P=\left\{P_{n}(x)\right\}$ be an arbitrary p.s. in $\pi$ and write for $n=0,1,2, \ldots$

$$
P_{n}(x)=\sum_{k=0}^{n}\left(\begin{array}{l}
n \\
k
\end{array}\right) \frac{(1+\alpha)_{n}}{(1+\alpha)_{k}} p_{n, k} x^{k} \quad\left(p_{n, n}=\beta_{n} \neq 0\right) .
$$

Let $L=\left\{L_{n}^{(\alpha)}(x)\right\}$ be the Laguerre p.s. defined by

$$
L_{n}^{(\alpha)}(x)=\sum_{k=0}^{n}\left(\begin{array}{l}
n \\
k
\end{array}\right) \frac{(1+\alpha)_{n}}{(1+\alpha)_{k}}(-x)^{k}, \quad n=0,1,2, \ldots
$$

Our problem is, therefore, to determine $p_{n, k}$ in (3.1) so that $P L=L P$.

In this section we prove our main theorem, shown here.

THEOREM. A.p.s. $P \in C_{\pi}\left(L^{(\alpha)}\right)$ if and only if

$$
p_{n, n-2 m-1}=\sum_{j=0}^{m} \frac{C_{2 j+1}}{2^{2 j+1}}\left(\begin{array}{r}
2 m+1 \\
2 j+1
\end{array}\right) \nabla^{2 j+1} P_{n, n-2 m+2 j}
$$


and

(b) $p_{n, n-2 k}$ are arbitrary with $p_{n, n} \neq 0$.

Here $\nabla$ is the backward difference operator acting on $n: \nabla f(n)=$ $f(n)-f(n-1)$.

Proof. We first note that $P L=L P$ is equivalent to requiring that for $j=0,1,2, \ldots, n$ and $n \geqq 0$ we have

$$
\sum_{k=j}^{n}(-1)^{k}\left(\begin{array}{l}
n-j \\
k-j
\end{array}\right) p_{k, j}=\sum_{k=j}^{n}(-1)^{j}\left(\begin{array}{l}
n-j \\
k-j
\end{array}\right) p_{n, k} .
$$

We next see that by putting $j=n, n-1$, etc. in (3.3) we get that $p_{n, n}$ is arbitrary, that $p_{n, n-1}=-(1 / 2)\left(\beta_{n}-\beta_{n-1}\right)$ for $n=1,2,3, \cdots$ so that (3.3) determines uniquely $p_{n, n-2 m-1}$, and that $p_{n, n-2 m}$ remains arbitrary.

To find the general solution of (3.3) we note that (3.3) can be rewritten in the form

$$
\sum_{k=0}^{s}(-1)^{k}\left(\begin{array}{l}
s \\
k
\end{array}\right) p_{n+k-s, n-s}=\sum_{k=0}^{s}\left(\begin{array}{l}
s \\
k
\end{array}\right) p_{n, n-k} \quad(0 \leqq s \leqq n)
$$

which implies, for $s=2 m(m=1,2, \cdots)$,

$$
\begin{aligned}
& \sum_{k=0}^{m-1}\left(\begin{array}{c}
2 m \\
2 k
\end{array}\right)\left\{p_{n-2 m+2 k, n-2 m}-p_{n, n-2 k}\right\} \\
= & \sum_{k=0}^{m-1}\left(\begin{array}{c}
2 m \\
2 k+1
\end{array}\right)\left\{p_{n-2 m+2 k+1, n-2 m}+p_{n, n-2 k-1}\right\},
\end{aligned}
$$

and for $s$ odd, $s=2 m+1$,

$$
\begin{aligned}
2 p_{n, n-2 m-1} & =\sum_{k=0}^{m}\left(\begin{array}{c}
2 m+1 \\
2 k
\end{array}\right)\left\{p_{n-2 m+2 k-1, n-2 m-1}-p_{n, n-2 k}\right\} \\
& -\sum_{k=0}^{m-1}\left(\begin{array}{c}
2 m+1 \\
2 k+1
\end{array}\right)\left\{p_{n-2 m+2 k, n-2 m-1}+p_{n, n-2 k-1}\right\} .
\end{aligned}
$$

We now show that (3.4) and (3.5) are satisfied if $p_{\nu, \nu-2 \mu}$ are arbitrary and

$$
p_{n, n-2 m-1}=\sum_{j=0}^{m}(-1)^{j-1} \alpha_{j}\left(\begin{array}{l}
2 m+1 \\
2 j+1
\end{array}\right) \nabla^{2 j+1} p_{n, n-2 m+2 j} .
$$

Indeed if we substitute (3.6) in the right hand side (RHS) of (3.4) we get

$$
\begin{aligned}
\mathrm{RHS}= & \sum_{k=0}^{m-1}\left(\begin{array}{c}
2 m \\
2 k+1
\end{array}\right) \sum_{j=0}^{k}(-1)^{j-1} \alpha_{j}\left(\begin{array}{c}
2 k+1 \\
2 j+1
\end{array}\right) \\
& \left\{\nabla^{2 j+1} p_{n-2 m+2 k+1, n-2 m+2 j+1}+\nabla^{2 j+1} p_{n, n-2 k+2 j}\right\} .
\end{aligned}
$$

Since $\nabla^{2 j+1} f(n)=\sum_{r=0}^{2 j+1}(-1)^{r}\left(\begin{array}{c}2 j+1 \\ r\end{array}\right) f(n-r)$ then the above expression (3.7) is a sum of terms of the form $p_{n-\mu, n-\mu-2 k}$. To show that (3.6) satisfies 
(3.4) we must show that the coefficient of $p_{n-\mu, n-\mu-z k}$ is $-\left(\begin{array}{l}2 m \\ 2 k\end{array}\right)$ if $\mu=0$, is $\left(\begin{array}{c}2 m \\ 2 k\end{array}\right)$ if $\mu=2 m-2 k$ and is zero if $\mu \neq 0$ or $\mu \neq 2 m-2 k$.

For example in the latter case, the coefficient of $p_{n-\mu, n-\mu-2 k}$ in (3.7) is a multiple of

$$
\sum_{j=0}^{m-k-1}(-1)^{j-1} \alpha_{j}\left\{\left(\begin{array}{c}
\mu \\
2 m-2 k-2 j-1
\end{array}\right)-\left(\begin{array}{c}
2 m-2 k-\mu \\
2 m-2 k-2 j-1
\end{array}\right)\right\}
$$

which is zero by Lemma 3 .

Similarly $\mu=0$ and $\mu=2 m-2 k$ follows from Lemma 1 .

Formula (3.5) can be seen to be satisfied by (3.6). This time we need to use Lemmas 1 and 2 and also we must show that that coefficient of $p_{n-\mu, n-\mu-2 k}$ is

$$
\sum_{j \geq 0}\left[\left(\begin{array}{c}
2 m-2 k+1-\mu \\
2 m-2 k-2 j
\end{array}\right)+\left(\begin{array}{c}
\mu \\
2 m-2 k-2 j
\end{array}\right)\right] \alpha_{j}(-1)^{j-1}=0 .
$$

This formula is a consequence of Lemma 2. This finishes the proof of the main theorem.

Formula 3.6 can be written operationally using the Euler polynomials $E_{n}(x)$. To do this let $\eta f(n, m)=f(n-1, m)$ and $\mu f(n, m)=f(n, m-1)$ so that $\nabla f(n, n)=(1-\eta \mu) f(n, n)$. We get

$$
p_{n, n-2 m-1}=(1-\eta \mu)^{2 m+1} E_{2 m+1}\left(\frac{\mu}{1-\eta \mu}\right) \cdot \beta_{n} .
$$

where we have again written $\beta_{n}=p_{n, n}$.

4. Special Cases. (a) $L^{(\alpha)}$ commutes with itself. This case follows when $p_{n, n-k}=(-1)^{n-k}$. Formula (3.6) can be seen to be satisfied since it implies that

$$
p_{n, n-2 m-1}=(-1)^{n}\left\{(1+C)^{2 m+1}-1\right\}=(-1)^{n-1} .
$$

This is easily seen because $(1+C)^{2 m+1}=0$ for $m=0,1,2, \cdots$.

(b) Let $\beta_{n}=p_{n, n}=n+\alpha$ and let $p_{n, n-2 k}=0$ for $k>0$. Then easy calculations show that

$$
P_{n}(x)=(n+\alpha) x^{n}-\frac{1}{2} n(n+\alpha) x_{n-1} .
$$

The commutativity implies the known recurrence formula for the Laguerre polynomials $\left.L_{n}^{(\alpha)}(x)-n L_{n-1}^{(\alpha)}(x)=L_{n}^{(\alpha-1)}\right)(x)$. The polynomial set $\left\{P_{n}(x)\right\}$ is not of the Boas-Buck type.

(c) The "symmetric subgroup" $\Sigma$. A p.s. $P$ is said to be symmetric if $P_{n}(-x)=(-1)^{n} P_{n}(x)$. It is easy to argue that the class of all symmetric p.s. $\Sigma$ with umbral composition forms a subgroup of $\pi$. We ask the question, what are the elements of $C_{\Sigma}\left(L^{(\alpha)}\right)$ ? 
To answer this question we note first that $P \in \Sigma \Rightarrow p_{n, n-2 m-1}=0$ for $m=0,1, \ldots,[n-1 / 2]$.

Putting $m=0$ in (3.6) shows that $p_{n, n}$ is independent of $n$. It now follows by induction on $m$ that $p_{n, n-2 m}=\gamma_{2 m}$ is independent of $n$. Thus such polynomial sets are given by

$$
P_{n}^{(r)}(x)=\sum\left(\begin{array}{c}
n \\
2 k
\end{array}\right) \frac{(1+\alpha)_{n}}{(1+\alpha)_{n-2 k}} \gamma_{2 k} x^{n-2 k}
$$

Furthermore one can easily show that $P_{n}^{(\gamma)}\left(P^{(\mu)}\right)=P_{n}^{(\mu)}\left(P^{(\gamma)}\right)=P_{n}^{(\delta)}(x)$ where $\delta_{2 n} /(2 n) !=\sum_{k}\left(\begin{array}{c}2 n \\ 2 k\end{array}\right) \mu_{2 k} \gamma_{2 n-2 k}$ so that we have the following result.

THEOREM. $C_{\Sigma}\left(L^{(\alpha)}\right)$ is a commutative subgroup of $C_{\pi}\left(L^{(\alpha)}\right)$.

We also remark that elements of $C_{\Sigma}\left(L^{(\alpha)}\right)$ are related to Brenke polynomials since we can show that

$$
\sum_{n=0}^{\infty} \frac{t^{n}}{n !(1+\alpha)_{n}} P_{n}^{(r)}(x)=\left(\sum_{n=0}^{\infty} \gamma_{2 n} \frac{t^{n}}{(2 n) !}\right) \cdot{ }_{0} F_{1}(-; 1+\alpha ; x t)
$$

where ${ }_{0} F_{1}(-; 1+\alpha ; u)=\sum_{n=0}^{\infty} u^{n} / n !(1+\alpha)_{n}$.

The case $r=\{1\}$ gives $P_{n}^{(1)}(x)=1 / 2\left\{(-1)^{n} L_{n}^{(\alpha)}(x)+L_{n}^{(\alpha)}(-x)\right\}$.

(d) As remarked earlier Appell showed that $\mathscr{A}$ is a subgroup of $\pi$. To determine $C_{\mathscr{A}}(L)$ we see that if $P \in \mathscr{A}$ then $P_{n}(x)=\sum\left(\begin{array}{l}n \\ k\end{array}\right) a_{n-k} x^{k}$. Hence $p_{n, n-k}=(1+\alpha)_{n-k} /(1+\alpha)_{n} a_{k}$ where $a_{k}$ is independent of $n$. Since ${ }_{n, n-2 k}$ are arbitrary so are $a_{2 k}$. Using (3.6) we can show that

$$
a_{2 m+1}=-(2 m) ! \sum_{k=0}^{m}\left(\begin{array}{c}
2 m+1 \\
2 k+1
\end{array}\right) \frac{C_{2 k+1}}{2^{2 k+1}} \frac{(2 m-2 k)}{(2 m-2 k) !} a_{2 m-2 k} .
$$

We can also show that such p.s. are generated by

$$
e^{E(\log (1-t))+x t}=\sum_{n=0}^{\infty} A_{n}(x) \frac{t^{n}}{n !}
$$

where $E(t)$ is an arbitrary even function of $t$.

\section{REFERENCES}

1. N. A. Al-Salam and W. A. Al-Salam, The Centralizer of the Laguerre Polynomial Set., Linear and Multilinear Algebra 7 (1979), 149-154.

2. P. Appell, Sur une classe de polynomes, Ann. Sci. Ecole Norm. Sup. (2) 9 (1880), 119-144.

3. R. P. Boas and R. C. Buck, Polynomials defined by generating relations, Amer. Math. Monthly 63 (1956), 626-632.

4. J. Goldberg and J. W. Brown, Generalized Appell connection sequences, J. Math. Analysis and Appl. 46 (1974), 242-248.

5. G. C. Rota, D. Kahaner and A. Odlysko, On the foundation of combinatorial theory VIII. Finite Operator Calculus, J. Math. Anal. and Appl. 42 (1973), 685-760. 
6. I. M. Sheffer, On sets of polynomials and associated linear functional operators and equations, Amer. J. Math. 53 (1931) 15-38.

The University of Alberta, Edmonton, Canada T6G 2G1 
